\title{
Análisis comparativo de la innovación de las empresas mexicanas y extranjeras en el sector farmacéutico (Comparative analysis of the innovation of mexican and foreign companies in the pharmaceutical sector)
}

\section{Pablo Alejandro Castro Rodríguez •}

\begin{abstract}
Pharmaceutical innovation is the basis of the health sector, since it constantly improves the quality of life of the human being. Schumpeter have influenced notably the theories of innovation, stating that economic development is driven by it, speaking of the dynamic process in which new technologies replace the old ones and that feed the process of change. On the other hand, the Research and Development (R\&D) process manages the returns on investment, allowing demonstrating how profitable the innovation process can be. In Mexico, the signs of innovation have remained on the side of foreign companies, while Mexican companies have settled for the majority of the generic market. This quantitative study collects data on the perception of experts in the pharmaceutical field, mostly students of the biotechnology pharmaceutical chemistry career at the Universidad del Valle de México, in Monterrey, Nuevo Leon, using surveys based on the OECD manuals for innovation and R\&D, comparing both the quality of innovation and the $R \& D$ process in Mexican companies and foreign companies. The perception of the students intuits the current context of the pharmaceutical industry in Mexico. The study shows a great relationship between the variables and a disparity of perception between foreign and Mexican companies.
\end{abstract}

Key words: pharmaceutical companies, pharmaceutical innovation, research and development (R\&D)

JEL: 0310, 0320.

\footnotetext{
- Universidad Autónoma de Nuevo León, Facultad de Ciencias Politicas y Relaciones Internacionales, Monterrey, N.L. México.Email: pablo.acrdz@gmail.com
} 
Resumen. La innovación farmacéutica es la base del sector salud, ya que permite mejorar constantemente la calidad de vida del ser humano. Schumpeter han influido notablemente en las teorías de la innovación, afirmando que el desarrollo económico esta movido por la misma, hablando del proceso dinámico en el cual nuevas tecnologías sustituyen a las antiguas y que alimentan el proceso del cambio. Por otra parte, el proceso de Investigación y Desarrollo $(l+D)$, maneja los rendimientos de la inversión, permitiendo demostrar que tan redituable puede llegar a ser el proceso de la innovación. En México, los indicios de innovación han permanecido del lado de las empresas extranjeras, mientras que las empresas mexicanas se han conformado con el mercado genérico en su mayoría. Este estudio cuantitativo recopila los datos de la percepción de conocedores de la materia farmacéutica, en su mayoría estudiantes de la carrera de químico farmacéutico biotecnólogo de la Universidad del Valle de México, en Monterrey, Nuevo León, utilizando encuestas basadas en los manuales de la OCDE para la innovación y la l+D, para comparar tanto la calidad de la innovación como el proceso de I+D de las empresas mexicanas y de las extranjeras. La percepción de los estudiantes intuye el contexto actual de la industria farmacéutica en México. El estudio muestra una gran relación entre las variables y una disparidad de la percepción entre las empresas extranjeras y mexicanas.

Palabras clave: empresas farmacéuticas, innovación farmacéutica, investigación y desarrollo $(I+D)$

\section{Introducción}

La industria farmacéutica de creación de medicamentos para uso humano es una de las industrias que se encuentran en constante movimiento, por lo cual requiere estar actualizada a los diversos momentos históricos para adaptarse a las necesidades de la sociedad, pues una industria farmacéutica sana, conlleva a una mejor calidad de vida para la sociedad. Una de las principales características de la industria farmacéutica es su actividad competitiva por el dinamismo que presentan en el sector de innovación y obtener mayores ganancias mediante el descubrimiento de nuevas moléculas, invirtiendo en investigación y desarrollo.

El análisis comparativo que se realizó, busca comprender las realidades de la industria farmacéutica en México en cuanto a las empresas de origen mexicano y las transnacionales, permitirá demostrar el contexto en el mercado de innovación, desde un punto de vista perceptivo, codificando los datos asignados a las modalidades observadas en base a un cuestionario creado, partiendo del marco referente al apoyo científico para medir las actividades científicas y tecnológicas y la interpretación de datos sobre la

\section{P. A. Castro}


innovación, elaborado por la OCDE, apoyado por estudiantes e investigadores de la Universidad del Valle de México (UVM).

La importancia de recabar información a los alumnos de licenciatura, radica en encontrar la percepción de posibles trabajadores de la industria, y conocer sus indicadores y estándares que tienen respecto a las empresas mexicanas y extranjeras de su futura área laboral.

México es un reflejo del mercado globalizado de la industria farmacéutica, presenta altos grados de inversión privada por parte de las empresas transnacionales, las cuales dominan el mercado de patentes vigentes en el país, mientras las empresas nacionales han quedado rezagadas en el campo de innovación.

Aunque es un mercado en constante crecimiento, presenta desigualdad en la competencia. Es notorio como los países desarrollados han obtenido las principales ventajas de una industria globalizada y se han adaptado a las normas de propiedad intelectual, viéndose beneficiados, mientras los países en desarrollo enfocan su mercado en productos genéricos, que les impide crecer en su capacidad de innovación y el cual se ha vuelto más estricto conforme el mercado lo requiere.

Uno de los puntos importantes de la industria farmacéutica global, es promover la innovación para seguir combatiendo las principales enfermedades que acusan las distintas sociedades en diferentes países. Por eso el objetivo de analizar los diversos beneficios de innovar un mercado globalizado.

\section{Innovación farmacéutica}

La competitividad en el entorno global representa un reto para las empresas transnacionales que buscan adaptar al entorno económico mundial, por lo que la innovación ha presentado un elemento esencial para el desarrollo y crecimiento de la empresa. La OCDE (2006) afirma que los agentes principales de la innovación deben ser las empresas, considerando relevante este aspecto, para el crecimiento de la economía interna. Schumpeter (citado en Ávila y Alarcón, 2014, p. 147) describe la innovación como "motor de desarrollo económico y un proceso dinámico continuo, donde las antiguas tecnologías son desplazadas por las nuevas". Este proceso incluye un manejo por parte de la industria de la I+D. 
Un nuevo producto 0 proceso puede colocar al innovador en una posición ventajosa en el mercado. La empresa puede obtener una ventaja competitiva, permitiendo aumentar la demanda y su margen de beneficio (OCDE, 2006, p. 38). Para la industria farmacéutica, la innovación es un motor que mueve el mercado, pues la falta de innovación produciría escases de medicamentos para las nuevas enfermedades que se producen en el mundo, así como nuevos tratamientos que mejoren la calidad de vida de la sociedad.

En el sector farmacéutico, la innovación se puede percibir como un ciclo, que parte de la I+D de compuestos básicos nuevos, pasa por los ensayos y el desarrollo de productos nuevos y alcanza la distribución de estos productos, para regresar a la $1+D$ de productos nuevos con una vigencia sistemática posterior a la entrada en el mercado y la creación de un modelo de demanda cada vez más eficaz basado en las necesidades de salud (OMS, OMPI, OMC, 2013).

Hay dos tipos de innovación farmacéutica aprobada por la Food and Drug administration (FDA) de Estados Unidos; primero las innovaciones novedosas que son nuevas moléculas; y las innovaciones incrementales que son nuevos fármacos creados modificando las moléculas existentes. Las innovaciones incrementales, también conocidas como innovaciones "continuas" o "acumulativas", representan una proporción creciente de la innovación y utilización farmacéutica. Las innovaciones incrementales pueden generar valor mediante: la creación de nuevos fármacos que utilizan las moléculas existentes para tratar diferentes enfermedades, cambiar la formulación química o el ingrediente activo de un fármaco para aumentar la eficacia del fármaco y reducir los efectos secundarios, crear fármacos combinados 0 reducir el número de pastillas 0 dosis, lo que aumenta la adherencia del paciente a un régimen de fármaco. (Hult, 2015).

Hablar de innovación es hablar de la creación de nuevas patentes, así como de I+D que forma parte esencial del proceso inventivo, y que cada vez, la posibilidad de invertir es más reducida para las empresas farmacéuticas, lo cual reduce el mercado de innovación global. Las patentes promueven innovación, proporcionando un incentivo para invertir en $I+D$, mientras que también funciona para estructurar, definir y construir asociaciones de innovación.

\section{P. A. Castro}


Las empresas innovadoras, que no necesariamente deben consistir en grandes empresas, sin embargo, es una constante actual, son quienes están mejor preparadas para materializar los beneficios de la I+D, con el desafío de reducir el tiempo invertido en producción y el ciclo de innovación, para poder garantizar mayor rentabilidad (Garabato, 2013). Las empresas innovadoras con altos activos de inversión privada, pueden financiar proyectos con mayor facilidad mediante la obtención de capital de riesgo. Los inversores consideran que los derechos exclusivos, son impulsores de rentabilidad y ventaja competitiva (Gawel, 2016). Y es que entre más grande es la empresa, menor es el impacto económico que puede recibir en caso de una inversión errónea, mientras que las medianas y pequeñas empresas no se pueden permitir esa clase de riesgos.

La reducción generalizada de la inversión en I+D es alarmante porque la industria farmacéutica requiere de una investigación intensiva y depende en gran medida de la innovación y desarrollo de nuevos medicamentos. Este proceso se ha hecho más caro a través de los años (Tang, 2013). La tendencia industrial de recortar los presupuestos de I+D, reduciendo los nuevos productos en las empresas innovadoras, significan una disminución en la innovación futura, lo cual produce una perdida para las economías nacionales, sin mencionar la afectación de salud en la población por no contar con los medicamentos requeridos.

La falta de incentivos significa la única razón detrás de la escasez de innovación farmacéutica. Se necesitan soluciones para reducir el alto costo del desarrollo de nuevos fármacos, así como nuevos modelos de I+D para mejorar la eficacia del sistema que enmarca la innovación. El sistema de patentes ha desempeñado tradicionalmente un papel indispensable para incentivar la innovación farmacéutica, y la actual falta de inversión sugiere una insuficiencia del sistema que debe arreglarse (Tang, 2013). Las mismas patentes han permitido a las empresas internacionales poder tener la certeza de invertir donde se considera que hay mayor seguridad en el tema.

Un sistema de patentes complejo, como el que presenta la industria farmacéutica, no ha permitido a las empresas pequeñas competir en igualdad de condiciones contra las transnacionales, lo que impide que el mercado de innovación tenga un mayor número de competidores, los cuales provienen en su mayoría de países desarrollados. 


\section{Investigación y desarrollo}

Para el proceso de innovación farmacéutica, la I+D forma parte compleja, pero esencial, para el descubrimiento de nuevos medicamentos que ayuden al dinamismo constante al cual se enfrenta el mercado global de la industria farmacéutica. Este proceso requiere la participación de una gran cantidad de investigadores, en diversas disciplinas científicas, donde se analizan diversos compuestos químicos, que puede llegar a tardar hasta 15 años desde la idea original del laboratorio a la aprobación del medicamento (Luengo. 2012). Las empresas requieren tanto el tiempo, como la inversión para poder general el proceso. A nivel internacional, los grandes laboratorios han desarrollado estrategias comerciales, a través de fusiones o absorciones de laboratorios más pequeños, para poder realizar avances en I+D que les permita asegurar el mercado.

La industria farmacéutica ha percibido en las últimas décadas una crisis de productividad, pues el gasto en $I+D$ ha crecido exponencialmente mientras que la innovación se ha mantenido relativamente estable. Estas tendencias se han percibido en Estados Unidos, ya que desde los años 50 el número de nuevas moléculas cayó un $60 \%$ mientras que el gasto en I+D aumento más de 280\% entre 1996 y 2010 (Hult, 2015). El aumento en los costos de I+D se atribuye en gran parte a que más candidatos a fármacos fallan en ensayos en etapas posteriores, al mayor costo de capital y a mayor rigidez regulatoria, hace que se requieran estudios más largos, antes de que un fármaco pueda ser aprobado (King, 2013).

El proceso de desarrollo de un nuevo fármaco se ve afectado por una serie de factores que repercuten en el costo de innovación. (Galdón, 1994, p.211) Se han comprometido a la investigación recursos determinantes para el descubrimiento de nuevas moléculas que permitan completar el ciclo de innovación, lo cual compromete la misma investigación, ya que para las empresas el riesgo de inversión puede llegar a presionar un medicamento que no tiene la calidad requerida para salir al mercado. Un nuevo fármaco se deriva de un proceso, que es difícil calcular en cuanto al costo, y por el cual Galdón (1994) menciona que es complicado dar un valor significativo "a los productos, sus gastos proporcionales de estructura, mantenimiento de instalaciones, servicios, etc., así como las inversiones en instalaciones y

\section{P. A. Castro}


aparatos, ya que parte de dichos recursos se utilizarán para el desarrollo de otras nuevas entidades químicas (p.224)".

Hablar de ganancias en cuanto a la I+D farmacéutica es complicado. Solo un pequeño porcentaje de las moléculas lanzadas se convierten en grandes éxitos económicos. La gran mayoría de los fármacos no cubren los costos medios de I+D, ni logran un éxito económico marginal. Las empresas invierten millones de dólares en I+D para obtener el derecho de una distribución monopólica del producto (Vernon, 2003). Es aquí donde las empresas transnacionales pueden permitirse tener riesgos económicos, en los cuales cuentan con mayor calidad del proceso de I+D.

La verificación independiente y la transparencia no se practican con frecuencia en la rama de I+D farmacéutica, por lo que el actual proceso está marcado por los fines lucrativos en lugar de las mejores prácticas en investigación científica. La investigación optima del proceso de I+D debe ser llevada a cabo entre la comunidad global de académicos alejada del proceso de investigación médica con fines de lucro (King, 2013) ya que puede llegar a perjudicar el proceso, pasando la investigación clínica a segundo plano por debajo de las ganancias que se buscan obtener.

Las empresas farmacéuticas han ampliado significativamente el número de sus propios empleados dedicados a la función de I+D. En su perfil de la industria y en sus informes anuales de encuestas durante varios años, PhRMA ha proporcionado información anual sobre el empleo en I+D de sus firmas miembro. Entre 1980 y 2000 , el empleo total en I+D aumentó a una tasa compuesta anual del $5,4 \%$, y el personal científico y profesional aumentó a una tasa anual del 7,4\% (DiMasi, Hansen y Grabowaki, 2003). Esto permite ampliar el margen de estructura, aunque las estadísticas representadas muestran el total de la industria farmacéutica y no solo de México, no varía tanto pues en un periodo de 2007 a 2013, los empleos se incrementaron $5.4 \%$, según información de la Cámara Nacional de la Industria Farmacéutica (CANIFARMA).

\section{Innovación farmacéutica en México}

La industria farmacéutica en México está llena de contrastes, por un lado existe la inversión por parte de grandes empresas transnacionales, que aprovechan un mercado en crecimiento, y por el otro las empresas 
mexicanas que han presentado dificultades para competir en la producción de medicamentos para uso humano. México se ha visto obligado a modificar su industria para adaptarse a los mercados internacionales que exigen mayores condiciones para la protección a los derechos de propiedad intelectual, incluyendo el mercado de las invenciones farmacéuticas, para dar garantías a la tutela de los derechos de patente a sus titulares (Ampudia, en el libro de Oropeza, 2012). Las empresas farmacéuticas extranjeras han generado una ganancia redituable desde la apertura comercial, ya que han aportado grandes inversiones tanto en el área de $I+D$ como en mercadotecnia y distribución de medicamentos. Esto ha demostrado la capacidad de México para soportar una industria farmacéutica sana, pero las empresas mexicanas siguen siendo productoras de genéricos.

El mercado de las empresas farmacéuticas mexicanas ha sido dinámico si se trata solo del nacional, pues han aprovechado la caducidad de patentes para incursionar en los medicamentos genéricos. González y Barraza (2011) mencionan que en el 2010 "el 95.6\% de los medicamentos consumidos en el mercado mexicano están fuera de patente... Aunque los medicamentos innovadores con patente vigente solo representan el $4.4 \%$ del volumen, en términos de valor equivalen a $20.4 \%$ de las ventas del mercado" (p. 45). Para valorar esta situación, la necesidad del desarrollo de un mercado tan redituable como el farmacéutico, se necesita incursionar en la medida que sea posible en la utilidad que presentan las ganancias netas, tanto de los medicamentos genéricos como de las nuevas patentes.

Guerrero y Gutiérrez (2011) destacan tres fases en la producción de conocimiento patentado de la industria farmacéutica en México:

- La primera, se extiende 1980 hasta 1990, resaltando el uso de la estrategia imitativa, en el que las empresas se benefician al no tener que esperar la expiración de la patente del producto original para traer su producto al mercado.

- La segunda, de 1991 a 1996, sería la fase pre ADPIC, caracterizada por la negociación y puesta en operación de los cambios legislativos, que afectarían el proceso tradicional de transferencia e innovación tecnológica de la industria.

- Y la tercera, sería la fase de los ADPIC, en la cual se homogenizan los DPI a nivel internacional (p.109). 
La balanza comercial para la industria farmacéutica en México, se ha visto desfavorecida para el mercado mexicano, pues ha crecido la desigualdad constantemente entre importaciones y exportaciones, mostrando la poca capacidad de las empresas farmacéuticas mexicanas de competir en el mercado extranjero, mientras las transnacionales han tenido mayores oportunidades de invertir en el país, como se demuestra en la Tabla 1.

Tabla 1. Balanza comercial

\begin{tabular}{cccc}
\hline Año & Exportaciones & Importaciones & Balanza Comercial \\
\hline $\mathbf{2 0 0 0}$ & 673,005 & $1,013,001$ & $-339,996$ \\
$\mathbf{2 0 0 1}$ & 886,711 & $1,259,226$ & $-372,515$ \\
$\mathbf{2 0 0 2}$ & 968,550 & $1,463,707$ & $-495,157$ \\
$\mathbf{2 0 0 3}$ & $1,033,014$ & $1,778,766$ & $-745,752$ \\
$\mathbf{2 0 0 4}$ & $1,265,572$ & $2,168,505$ & $-902,933$ \\
$\mathbf{2 0 0 5}$ & $1,257,250$ & $2,435,047$ & $-1,177,792$ \\
$\mathbf{2 0 0 6}$ & $1,224,784$ & $3,024.321$ & $-1,799,787$ \\
$\mathbf{2 0 0 7}$ & $1,311,483$ & $3,388,983$ & $-2,077,500$ \\
$\mathbf{2 0 0 8}$ & $1,310,739$ & $4,071,420$ & $-2,760,681$ \\
\hline
\end{tabular}

Nota: Fuente: González y Barraza (2012) con datos del banco de México

Las empresas transnacionales han sabido aprovechar el mercado mexicano, sin embargo, esto ha rezagado a las empresas nacionales. La innovación en el sector farmacéutico por parte de las empresas nacionales mexicanas ha sido reducida desde la apertura comercial mexicana. Gerardo Enríquez en el libro de Becerra (2013) menciona que "desde 1993 se han eliminado las restricciones a la inversión extranjera directa en el sector farmacéutico, aunque desde 1986 la industria se somete a desgravación, por lo que los aranceles promedio disminuyeron de $24.2 \%$ en 1980 , a $12.7 \%$ en $1998 "$ (p. 124). Esto ha permitido a México ser considerado como una importante plataforma para las empresas transnacionales. Desde 1991, cuando la ley mexicana admitió en el estatuto de patentables a los farmacéuticos, se incrementaron de manera sustancial las solicitudes, aunque en este sector la gran mayoría fue de empresas no nacionales, lo cual se observa como una tendencia que se extiende hasta la actualidad (Ampudia en el libro de Oropeza y Guizar, 2012, p. 457). En este año se crea 
la nueva Ley de Propiedad Industrial. Y como se muestra en la Figura 1 los primeros años en vigor de esta ley las patentes fueron otorgadas casi en su totalidad a empresas extranjeras.

Figura 1. Patentes otorgadas según su origen

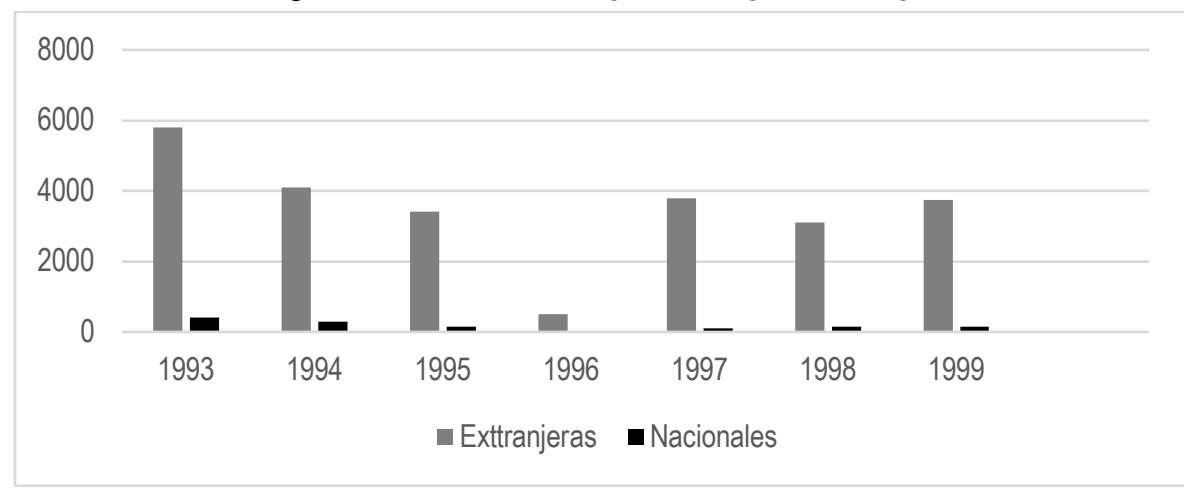

Nota: Fuente: García Saucedo, citada en Leyva, R. Wirtz, V. Dreser, A. \& Reich, M. (2006).

Una de las regiones comerciales más competitivas a nivel mundial es la que componen México, Estados Unidos y Canadá, a través de lo que ha sido el aprovechamiento del Tratado de Libre Comercio de América del Norte (TLCAN). En materia farmacéutica, las empresas han desarrollado un mercado rentable, por las facilidades que ofrece el área económica. Conforme aumentaron las relaciones comerciales entre los países de América del Norte, se incrementó la presencia de las patentes extranjeras de productos farmacéuticos en México (Guerrero y Gutiérrez, 2011, p. 93) por lo cual la balanza comercial se inclinó a favor de las importaciones, perjudicando a las exportaciones por parte de las empresas mexicanas.

Los derechos de propiedad intelectual tuvieron un papel importante en las rondas de negociación, mientras la industria farmacéutica estadounidense hacía un esfuerzo importante por incrementar los estándares en la misma área (Harrison, 2001). Las empresas farmacéuticas siempre han sido de gran relevancia al momento de enfocarse en la cuestión de propiedad intelectual, sobre todo las transnacionales que tienen un gran poder adquisitivo.

El artículo 1711 del TLCAN establece una norma sobre exclusividad que exige a todos los países signatarios un periodo mínimo de exclusividad de cinco años a partir de la fecha de aprobación de la comercialización del 
producto (Correa, 2002, p. 12) lo que ha permitido principalmente a la industria farmacéutica estadounidense posicionarse en el mercado, así como a las demás empresas extranjeras poder tener una participación estratégica que les permita tener mayores ganancias. Tras el TLCAN las empresas farmacéuticas de Estados Unidos y Canadá se han consolidado en el mercado de innovación, y como grandes exportadoras, mientras las empresas mexicanas han reducido su participación en el proceso de innovación, ya que solo produce bienes finales (Guerrero y Gutiérrez, 2011).

La tasa de fracaso es alta al invertir en innovación farmacéutica, se calcula que alrededor del $50 \%$ de las patentes solicitadas son rechazadas en México, según cifras del Instituto Mexicano de Propiedad Intelectual (IMPI) en un periodo de 15 años. Lo cual impide recuperar la inversión, así como generar beneficios sociales con la incursión de nuevos medicamentos. Las empresas innovadoras, ofrecen eficacia y seguridad en base a costosas actividades para I+D, en los cuales se calcula el costo medio de desarrollo de una nueva molécula es de alrededor de 600 millones de dólares, el cual tarda entre siete y diez años. Su recuperación y reinicio del ciclo son el principal argumento de las grandes empresas farmacéuticas para fortalecerse en la escala mundial en un sistema de patentes (Salomón, 2006). El sistema de patentes concede un derecho exclusivo con valor económico por un tiempo limitado a cambio de la divulgación tecnológica, lo cual funciona como una herramienta para la gobernanza de las empresas en un mercado de conocimiento global como lo es el farmacéutico (Gawel, 2016).

El acuerdo sobre Aspectos de los Derechos de Propiedad Intelectual relacionados con el Comercio (ADPIC) tuvo un impacto fundamental en el mercado de los medicamentos, ya que obligó a los países miembros a proporcionar patentes a partir de 2005 para todos aquellos procesos nuevos, que integraran alguna innovación que pudiera aplicarse en el terreno industrial (Mizrahi, 2010, p.18). El acuerdo intenta solucionar el problema mundial que existe con el reconocimiento de la patente, aunque sigue teniendo problemas en países como China. Después de la suscripción a los ADPIC, las patentes otorgadas en México aumento exponencialmente, aunque como se mencionó anteriormente, la mayoría de esas patentes eran solicitadas por empresas extranjeras. 
En la Tabla 2 se puede observar como incrementó el mercado de patentes en México durante el periodo de 1980 a 2007, aunque disminuyendo también el porcentaje de solicitadas-otorgadas:

Tabla 2. Mercado de patentes, periodo 1980-2007

\begin{tabular}{lccc}
\hline Fase & Periodo & Solicitadas & Otorgadas \\
\hline Imitativa & $1980-1990$ & 90 & 89 \\
Pre ADPIC & $1991-1996$ & 5,200 & 3,022 \\
ADPIC & $1997-2007$ & 26,869 & 12,933 \\
\hline
\end{tabular}

Fuente: Gutiérrez Castro y Gutiérrez R. (2011) con datos de SIGMA-IMPI

Para las empresas farmacéuticas han sido importantes las regulaciones internacionales en materia de propiedad intelectual, lo cual han adquirido como incentivo de innovación en los distintos países en los cuales tienen inversiones privadas.

\section{Hipótesis}

La hipótesis de esta investigación se presenta a continuación: $\mathrm{H} 1=$ Las empresas farmacéuticas extranjeras logran una mayor calidad en innovación al incrementar su capacidad en el proceso de I+D.

Las teorías cercanas a la hipótesis, en este caso la teoría de la innovación como causa del desarrollo de Schumpeter quien considera el proceso de producción como una combinación de fuerzas productivas, las que, a su vez, están compuestas por fuerzas materiales y fuerzas inmateriales. Las fuerzas materiales las componen los llamados factores originales de la producción (medios de producción producidos). Las fuerzas inmateriales las componen los "hechos técnicos" y los "hechos de organización social", que, al igual que los factores materiales, también condicionan la naturaleza y el nivel del desarrollo económico (Schumpeter, 1978).

\section{Variable dependiente}

El proceso para medir la calidad de la innovación farmacéutica, en cuanto a la percepción de expertos en la materia, se encuentra basada en el Manual

\section{P. A. Castro}


de Oslo, donde la recogida y análisis de datos sobre la innovación se basa en los conocimientos teóricos, así como la experiencia práctica adquirida efectuadas a nivel internacional, proponiendo directrices en cuanto a los elementos claves, que permite obtener resultados comparables (OCDE, 2006). El alcance del manual, en su tercera edición, se ocupa solamente del sector empresarial, midiendo el nivel de las empresas en innovación para cada sector, en este caso específico, el sector salud a través de la industria farmacéutica.

En este estudio, solamente se enfocara en la innovación del producto, o la creación de nuevas moléculas para medicamentos de uso humano. Las principales características a medir, que desarrolla el manual, son la calidad del proceso, la capacidad de ensayo, la capacidad de innovación de nuevas moléculas, la innovación incremental, los incentivos de creación de nuevas patentes, la redituabilidad que produce la innovación, y el nivel general de México en cuanto a innovación.

\section{Variable independiente}

La I+D pretende mostrar los cambios que presenta la innovación, cuando se invierte en el proceso de I+D, el cual recurre a las dimensiones a medir, señaladas en el Manual de Frascati elaborado por la OCDE para medir el proceso de I+D de las empresas en un sector determinado, que son el personal capacitado, los gastos, la calidad de los medios materiales, y como utilidad general, los esfuerzos nacionales en la materia. La capacitación del personal dedicado a la I+D, ofrece un medio concreto para medir los recursos dedicados, el interés general del personal, así como la capacidad científica de los mismos, constituyendo el elemento de recursos humanos.

Los indicadores a medir son la capacitación del personal, la calidad de la educación universitaria a la que acudieron, el personal de apoyo y utilización de personal universitario. Los gastos de I+D es la medida básica que comprende los gastos correspondientes a las actividades enfocadas a realizar el proceso (OCDE, 2003). La I+D es un proceso que requiere importantes inversiones, para poder desarrollar productos de mayor calidad. Los gastos manejan los indicadores de inversión, gastos en producción, y recursos públicos y privados. Los medios materiales denotan la eficacia del material utilizado para el proceso de I+D así como la capacidad y poder de 
uso de la empresa (OCDE, 2003). Los indicadores que componen los medios materiales, son el poder de compra, calidad de los recursos materiales, servicios de seguridad, calidad del área de trabajo, equipo para preparación y realización de pruebas, equipo tecnológico, adquisición de libros y publicaciones y la calidad de la biblioteca. Los recursos nacionales, miden la relación directa con la economía nacional, mostrando los efectos de la política científica, en cuanto a una percepción en conjunto, en este caso del sector farmacéutico (OCDE, 2003). Esta dimensión está compuesta por los indicadores de destinación de los gastos internos a la I+D y los esfuerzos nacionales en I+D en materia farmacéutica.

\section{Método}

El estudio es un diseño correlacional-causal, una vez estudiadas las variables perceptivas de las empresas mexicanas y extranjeras por parte de los estudiantes afines a la materia de innovación farmacéutica, buscando determinar una relación causa efecto entre la I+D para poder comparar los niveles de innovación. Además se pretende asociar las variables mediante un patrón predecible (Hernández, Fernández y Baptista, 2010) permitiendo analizar cómo influye el proceso de I+D de las empresas en su capacidad para innovar.

El instrumento a aplicar, para medir la percepción de la industria farmacéutica en cuanto a la capacidad de innovación y el proceso de I+D es la encuesta, que consiste en un método de recolección de datos que se realiza mediante un cuestionario aplicado a un determinado grupo de personas (Landero y González, 2009). El cual es útil en la investigación para medir la percepción de las empresas mexicanas y extranjeras.

El método de medición utilizado es la escala Likert, la cual tiene un nivel ordinal, que busca asignar un valor numérico a las variables, explicando el fenómeno producido en sector farmacéutico numéricamente. La codificación de los datos tiene como propósito asignar números a las modalidades observadas 0 registradas de las variables que constituyen la base de datos, así como asignar código (valor numérico) a los valores faltantes (aquellos que no han sido registrados u observados) (Landero y González, 2009). 
Las unidades de las que se extrajeron las encuestas son de la Universidad del Valle de México, en Monterrey, Nuevo León, en la carrera de licenciatura en químico farmacéutico biólogo, siendo cercana al objeto de estudio que es la innovación farmacéutica. Se realizaron 48 encuestas en una carrera que cuenta con 52 alumnos, con un nivel de confianza de $95 \%$ y un margen de error de $4 \%$ para determinar la fiabilidad del estudio con conocedores de la materia. El instrumento que se les aplico a los estudiantes genera resultados perceptivos de la situación en los laboratorios farmacéuticos, haciendo énfasis sobre la situación general en México y no solamente en el área de su localidad.

Además, se realizaran en referencia a unidades estadísticas, buscando la precepción de las empresas que innovan en el mercado de producción de medicamentos para uso humano, haciendo una separación de las empresas farmacéuticas mexicanas y las extranjeras. El estudio requiere que la población tenga características comunes, por lo cual los estudiantes están delimitados por el problema y objetivos de la investigación, referente a la innovación farmacéutica en cuanto a medicamentos para uso humano. El grupo seleccionado intentará generalizar los resultados en cuanto a la percepción de capacidad de innovación y el proceso de I+D, por una parte de las empresas mexicanas y por otra parte a las empresas extranjeras. Aunque no cuentan con una extensa experiencia laboral en el campo farmacéutico, los estudiantes presumen de un conocimiento susceptible que les permite idear el campo industrial.

Generalmente, las encuestas sobre innovación son sondeos sobre muestras aleatorias, en este caso el muestreo aleatorio simple, basada en el tamaño de población que trabaja actualmente en el sector farmacéutico de producción de medicamentos para uso humano. El análisis de fiabilidad en cuanto a la variable de innovación muestra que el Alfa de Cronbach es de .868 y para la I+D es .912, lo cual demuestra un modelo de consistencia interna, basado en el promedio de las correlaciones entre los indicadores.

\section{Reporte de resultados}

La variable de innovación mostró incidencia en las empresas farmacéuticas extranjeras (véase Tabla 3), un mayor potencial en cuanto a su calidad del proceso de innovación $(\mathrm{M}=3.77, \mathrm{DE}=.951)$ que se ve representado en las 
demás características como capacidad de ensayo $(\mathrm{M}=4.21, \mathrm{DE}=.771)$, innovación de nuevas moléculas $(M=4.21, D E=.824)$ e innovación incremental $(\mathrm{M}=3.98, \mathrm{DE}=.812)$, en comparación con las empresas mexicanas que en ninguna de estas categorías rebasan la media de 3 puntos.

La percepción de la calidad de innovación de las empresas mexicanas se encuentra por debajo de la media $(\mathrm{M}=2.85, \mathrm{DE}=.772$ ) superadas casi un punto por las empresas extranjeras.

Los aspectos generales como el incentivo de creación de nuevas patentes, la redituabilidad de las mismas o el nivel en general de las empresas en México, mostraron una media por debajo de los 3 puntos, que se compara con el pobre desempeño de las mismas empresas mexicanas en el mercado de innovación farmacéutica que perciben los estudiantes de farmacéutica.

Tabla 3. Descriptivos Innovación

\begin{tabular}{|c|c|c|c|c|c|}
\hline Variable & $\mathbf{N}$ & Mínimo & Máximo & Media & $\begin{array}{l}\text { Desviación } \\
\text { estándar }\end{array}$ \\
\hline $\begin{array}{l}\text { Calidad del proceso para innovar empresa } \\
\text { mexicana }\end{array}$ & 48 & 2 & 5 & 2.85 & .772 \\
\hline $\begin{array}{l}\text { Calidad del proceso para innovar empresa } \\
\text { extranjera }\end{array}$ & 48 & 2 & 5 & 3.77 & .951 \\
\hline $\begin{array}{l}\text { Capacidad de ensayo y desarrollo empresa } \\
\text { mexicana }\end{array}$ & 48 & 2 & 5 & 3.00 & .799 \\
\hline $\begin{array}{l}\text { Capacidad de ensayo y desarrollo empresa } \\
\text { extranjera }\end{array}$ & 48 & 3 & 5 & 4.21 & .771 \\
\hline $\begin{array}{l}\text { Capacidad de innovación de nuevas moléculas } \\
\text { empresa mexicana }\end{array}$ & 48 & 1 & 5 & 2.79 & 1.010 \\
\hline $\begin{array}{l}\text { Capacidad de innovación de nuevas moléculas } \\
\text { empresa extranjera }\end{array}$ & 48 & 2 & 5 & 4.21 & .824 \\
\hline $\begin{array}{l}\text { Capacidad de innovación incremental empresa } \\
\text { mexicana }\end{array}$ & 48 & 1 & 5 & 2.92 & .942 \\
\hline $\begin{array}{l}\text { Capacidad de innovación incremental empresa } \\
\text { extranjera }\end{array}$ & 48 & 2 & 5 & 3.98 & .812 \\
\hline Incentivo de creación de nuevas patentes & 48 & 1 & 5 & 2.71 & 1.129 \\
\hline Redituabilidad de nuevas patentes & 48 & 1 & 5 & 2.71 & .988 \\
\hline Nivel de México en innovación farmacéutica & 47 & 1 & 4 & 2.66 & .867 \\
\hline $\mathrm{N}$ válido (por lista) & 47 & & & & \\
\hline
\end{tabular}

Nota. Fuente: Elaboración propia utilizando el software SPSS 
Las empresas mexicanas poseen ligeramente mayor percepción en cuanto a su capacidad de I+D en general $(M=2.94$, $D E=.932)$ que al momento de innovar (véase Tabla 4), aunque sigue siendo considerablemente menor que la capacidad de las empresas extranjeras en México ( $\mathrm{M}=4.17, \mathrm{DE}=694)$, las cuales también aumentan la misma percepción contra su capacidad de innovación considerablemente.

Tabla 4. Descriptivos $1+D$

\begin{tabular}{lccccc}
\hline Variable & N & Mínimo & Máximo & Media & Desviación estándar \\
\hline Nivel de I+D de una empresa mexicana & 48 & 1 & 5 & 2.94 & .932 \\
Nivel de I+D de una empresa extranjera & 48 & 2 & 5 & 4.17 & .694 \\
N válido (por lista) & 48 & & & & \\
\hline
\end{tabular}

Nota. Fuente: Elaboración propia utilizando el software SPSS

El personal utilizado por las empresas mexicanas se encuentra menos capacitado $(\mathrm{M}=2.92, \mathrm{DE}=.794)$ que el personal de las empresas extranjeras $(\mathrm{M}=3.96, \mathrm{DE}=.798)$ como se muestra en la Tabla 5 y aunque la calidad universitaria del mismo personal se encuentra por encima de la media $(\mathrm{M}=3.49, \mathrm{DE}=.882$ ) las empresas de innovación farmacéutica en México no utilizan en menor medida el apoyo de estudiantes para la materia de $I+D(M=$ 3.13, DE = 1.084).

Tabla 5. Descriptivos Personal I+D

\begin{tabular}{llcccc}
\hline & N & Mínimo & Máximo & Media & Desviación estándar \\
\hline Capacitación del personal empresa mexicana & 48 & 1 & 5 & 2.92 & .794 \\
Capacitación del personal empresa extranjera & 48 & 1 & 5 & 3.96 & .798 \\
Calidad de la educación universitaria & 47 & 2 & 5 & 3.49 & .882 \\
Personal de apoyo & 48 & 2 & 5 & 3.13 & .815 \\
Frecuencia de utilización de personal de & 48 & 1 & 5 & 3.13 & 1.084 \\
universitario & & & & & \\
N válido (por lista) & 47 & & & & \\
\hline
\end{tabular}

Nota. Fuente: Elaboración propia utilizando el software SPSS

Los gastos en I+D, es una de las dimensiones más desequilibradas (véase Tabla 6), pues las empresas mexicanas presentan a penas una media de 2.44 (DE = .873) y la de las empresas extranjeras manejan un 4.19 (DE = .762). Los estudiantes perciben los recursos recibidos solamente en 2.77 (DE $=1.057$ ) lo cual permite apreciar como las universidades se encuentran alejadas del sector privado. 
Tabla 6. Descriptivos Gastos 1+D

\begin{tabular}{lcccccc}
\hline \multicolumn{1}{c}{ Variable } & N & Mínimo & Máximo & Media & Desviación estándar \\
\hline Capacidad de inversión empresa mexicana & 48 & 1 & 4 & 2.44 & .873 \\
Capacidad de inversión empresa extranjera? & 48 & 2 & 5 & 4.19 & .762 \\
Gastos económicos al momento innovar & 48 & 1 & 5 & 3.27 & 1.125 \\
Recursos privados o públicos que reciben & 48 & 1 & 5 & 2.77 & 1.057 \\
N válido (por lista) & 48 & & & & \\
\hline
\end{tabular}

Nota. Fuente: Elaboración propia utilizando el software SPSS

La calidad de los recursos materiales, poseen gran importancia en el proceso de $I+D$, pues a través de ellos se logran concretar los productos innovadores, permite a las empresas extranjeras manejar en su mayoría el mercado de innovación, mostrando un alto nivel de aprobación $(M=4.17$, DE $=.859$ ) mientras que las empresas mexicanas manejan productos de menor calidad ( $\mathrm{M}=2.92$, $\mathrm{DE}=1.069)$ un poco por debajo del valor medio (véase Tabla 7).

Tabla 7. Descriptivos Materiales $1+D$

\begin{tabular}{|c|c|c|c|c|c|}
\hline Variable & $\mathbf{N}$ & Mínimo & Máximo & Media & Desviación estándar \\
\hline $\begin{array}{l}\text { Poder de compra de recursos materiales } \\
\text { empresa mexicana }\end{array}$ & 48 & 1 & 5 & 2.92 & .895 \\
\hline $\begin{array}{l}\text { Poder de compra de recursos materiales } \\
\text { empresa extranjera }\end{array}$ & 48 & 2 & 5 & 4.06 & .885 \\
\hline $\begin{array}{l}\text { Calidad de recursos materiales empresa } \\
\text { mexicana }\end{array}$ & 48 & 1 & 5 & 2.92 & 1.069 \\
\hline $\begin{array}{l}\text { Calidad de recursos materiales empresa } \\
\text { extranjera }\end{array}$ & 48 & 2 & 5 & 4.17 & .859 \\
\hline $\begin{array}{l}\text { Servicios de seguridad para la } \\
\text { prevención de accidentes }\end{array}$ & 48 & 1 & 5 & 3.42 & 1.069 \\
\hline Área de trabajo e instalaciones & 48 & 1 & 5 & 3.23 & .994 \\
\hline $\begin{array}{l}\text { Equipo para preparación de materiales y } \\
\text { realización de experimentos, pruebas y } \\
\text { análisis }\end{array}$ & 48 & 1 & 5 & 3.02 & .887 \\
\hline Equipo tecnológico & 48 & 1 & 5 & 2.94 & .885 \\
\hline $\begin{array}{l}\text { Adquisición de libros, publicaciones } \\
\text { periódicas y anuarios }\end{array}$ & 48 & 1 & 5 & 2.85 & .989 \\
\hline $\begin{array}{l}\text { Calidad bibliotecaria } \\
\mathrm{N} \text { válido (por lista) }\end{array}$ & $\begin{array}{l}48 \\
48\end{array}$ & 1 & 5 & 3.06 & 1.019 \\
\hline
\end{tabular}

Nota. Fuente: Elaboración propia utilizando el software SPSS

En otros campos, como la calidad del área de trabajo ( $\mathrm{M}=3.23$, $\mathrm{DE}$ $=.994)$ y los servicios de seguridad $(M=3.42, D E=1.069)$, además de la calidad de la biblioteca con libros especializados $(M=3.06, D E=1.019)$ las

\author{
P. A. Castro
}


empresas mexicanas superan el estándar medio de 3 puntos, sin embargo, siguen sin acercarse al promedio de los 4 puntos, donde parecen estar estancadas. Los medios más importantes en el proceso de I+D que son los tecnológicos también se encuentran por debajo de la media $(M=294 \mathrm{DE}=$ 885).

La percepción de los recursos nacionales dedicados a la $I+D$ en materia farmacéutica es reflejo del pobre desempeño de las empresas mexicanas que no cuentan con el capital financiero de las empresas extranjeras (véase Tabla 8), pues los esfuerzos nacionales no alcanzan los 3 puntos $(\mathrm{M}=2.42 \mathrm{DE}=.919)$ y por su parte la destinación de recursos nacionales a la I+D presenta una media de 2.25 (DE = 1.082).

Tabla 8. Descriptivos Recursos Nacionales I+D

\begin{tabular}{lccccc}
\hline \multicolumn{1}{c}{ Variable } & N & Mínimo & Máximo & Media & Desviación estándar \\
Destinación de gastos internos para I+D & 48 & 1 & 5 & 2.25 & 1.082 \\
por parte del gobierno mexicano & & & & & \\
Esfuerzos nacionales & 48 & 1 & 4 & 2.42 & .919 \\
N válido (por lista) & 48 & & & & \\
\hline
\end{tabular}

Nota. Fuente: Elaboración propia utilizando el software SPSS

\section{Discusión de los resultados}

El proceso de I+D que incluye, el personal, los gastos, materiales y recursos nacionales, está íntimamente ligado a la calidad de innovación y funcionalidad para poder crear nuevos medicamentos. Es notorio como la I+D tiene un alcance con sus dimensiones que permite distinguir lo complicado que es para las empresas mexicanas el competir con las empresas extranjeras en los distintos factores.

Los laboratorios transnacionales logran traducir la complejidad del proceso de I+D en ganancias a través de la innovación. Desde la calidad y capacitación de su personal que logra contar con mayores facilidades, como mejores materiales y más recursos tecnológicos, por lo que ofrecen mejores ofertas laborales. Para las empresas mexicanas los recursos son limitados y su capacidad de I+D se limita a procesos repetitivos que terminan en la fabricación de productos genéricos. La balanza comercial es bastante notoria en cuanto al poderío de las empresas transnacionales, y la percepción de los 
estudiantes de farmacéutica no dista mucho de la complejidad de la teoría, que demuestra que las empresas mexicanas están rezagadas en los principales aspectos y que su capacidad de innovar es limitada o nula en un mercado dominado por empresas extranjeras.

Uno de los principales factores que impiden a las empresas farmacéuticas invertir en innovación es alto porcentaje de fracaso, ya que más de la mitad de patentes solicitadas no son aceptadas, las empresas extranjeras que muestran gran capacidad de inversión en contraste con las mexicanas, en una de los indicadores más disparejos del estudio, tienen esa capacidad de soportar los riesgos.

El desarrollo económico del sector es importante, ya que el nivel de México que se percibe en $I+D$, es comparable con los niveles que se perciben de las propias empresas mexicanas en el sector farmacéutico. México, con base en la teoría, no es uno de los países que más invierten en I+D, y la mayoría de las empresas extranjeras que si tienen capacidad inventiva y tienen participación en el mercado mexicano, son de países desarrollados, los cuales están ligados con el proceso de I+D.

La adaptación de México a los mercados internacionales, apegándose a la normatividad internacional, han permitido a las empresas extranjeras invertir con mayor confianza en el mercado mexicano, abriendo laboratorios dedicados a la producción de nuevos medicamentos, aunque la percepción en el estudio, muestra que a comparación del proceso de I+D, donde las empresas extranjeras están cercanas a la excelencia, recae un poco en la calidad de innovación, aunque siguen estando por encima de la media.

Como se mencionó en la teoría, uno de los aspectos más importantes de la innovación es la patente, el cual es un incentivo para innovar, y aunque Correa (2002) y Gutiérrez y Castro (2011) mencionan la rigidez de la patente en México, que para las empresas mexicanas ha resultado contradictorio ya que no tienen el nivel para invertir y desarrollar nuevas patentes, el incentivo de creación de nuevas patentes se encuentra por debajo de la media según la percepción estadística, así como la redituabilidad de nuevas patentes, lo cual hace suponer que el conocimiento sobre propiedad intelectual en nuestro país no está bien difundido.

En general el nivel de innovación farmacéutica de México también se encuentra por debajo de la media, y se percibe tan bajo como los demás niveles medidos, tanto de las empresas mexicanas como de los niveles del

\section{P. A. Castro}


gobierno mexicano para invertir en innovación, y aunque México tenga empresas farmacéuticas transnacionales no son consideradas a la par con la capacidad del país en general.

\section{Conclusión}

El incrementar la capacidad en el proceso de I+D definitivamente influye en la calidad de la innovación en la industria farmacéutica, y en la percepción de las empresas extranjeras, que demuestran tener un alto índice de $1+D$ en todas las dimensiones, y que demuestran por lo tanto calidad en la innovación y su competitividad es más alta.

Por otra parte las empresas mexicanas perciben un bajo nivel en el proceso de $I+D$, y su capacidad de innovación está por debajo de la media, por lo que un mercado tan competitivo como lo es el farmacéutico, se rezagan en el campo de innovación, dedicando la mayor parte de sus inversiones al mercado genérico.

Las desventajas que tienen las empresas mexicanas contra las extranjeras, deben ser estudiadas en mayor medida por el gobierno mexicano, quien también presenta un bajo índice de aprobación en ambas variables, influyendo más en la inversión pública para el desarrollo de estudios científicos con universidades que permitan relacionar más al sector privado, sobre todo a las empresas mexicanas, con el sector público. Si se mejora en los aspectos fundamentales de la I+D, tanto del personal, como los gastos, y los materiales, y aunque no dependa de las empresas, también los recursos nacionales destinados a la $I+D$, es factible mejorar la capacidad de innovación, medible en una mayor producción de patentes vigentes.

\section{Referencias}

Ampudia, S. (2012). Innovación, industria farmacéutica y mercado. El caso México. En Oropeza, A. (Ed.) Los retos en la industria farmacéutica en el siglo XXI. Una visión comparada sobre su régimen de propiedad intelectual. Ciudad de México: UNAM, Instituto de Investigaciones Jurídicas.

Ávila, L. \& Alarcón, J. (2014). Factores críticos de éxito de las empresas innovadores: una investigación documental, Innovaciones de negocios UANL, 11(21),143-67. 
Correa, C. (2002). Protección de los datos presentados para el registro de productos farmacéuticos. Implementación de las normas del acuerdo TRIPS. Buenos Aires: Centro del Sur.

DiMasi, J. Hansen, R. \& Grabowski, H. (2003). The price of innovation: new estimates of drugs development costs, Journal of Health Economics, 22, 141-185.

Enríquez. G. (2013). El impacto de tratados internacionales en salud pública y la industria farmacéutica en México. En Becerra, M. (Coord.). Propiedad intelectual y farmacéuticos. Hacia una política de Estado. Ciudad de México: UNAM, Instituto de Investigación.

Harrison, C. (2001) How the U.S. research pharmaceutical industry snatched international victory from the jaws of domestic defeat (Tesis de Doctorado). University of North Carolina.

Galdón, C. (1994). Innovación y progreso en la industria farmacéutica (perspectiva española y mundial) (Tesis de doctorado). Universidad Complutense de Madrid.

Garabato, F. (Ed) (2013). Laboratorios e industria farmacéutica. Reporte sectorial. Buenos Aires: Bdoargentina.

Gawel, C. R. (2016). Patent protection as a key driver for pharmaceutical innovation, Pharmaceuticals Policy \& Law, 18(1-4), 45-53.

González, E. \& Barraza, M. (2011). Trabajando por la salud de la población: Propuestas de política para el sector farmacéutico. Versión para el dialogo. Ciudad de México: Funsalud.

Guerrero, R. \& Gutiérrez, R. (2011). Los ADPIC y el TLCAN en la industria farmacéutica mexicana: Un análisis TradeCAN, Economía: teoría y práctica, 35, 93-129.

Hernández, R., Fernández, C. \& Baptista, M. (2010). Metodología de la investigación. Ciudad de México: McGraw Hill.

Hult, K. (2015). Incremental innovation and pharmaceutical productivity (Tesis de doctorado). University of Chicago.

King, T. (2013) Pharmaceutical research and development. A key informant assessment of whether an "open-science" model could improve clinical research in terms of quality and efficiency (Tesis de doctorado). University of North Carolina.

Landero, R. \& González, M. (2009) Estadística con SPSS y metodología de la investigación. Monterrey: Trillas.

Leyva, R., Wirtz, V., Dreser, A. \& Reich, M. (2006). Hacia una política farmacéutica integral para México, Salud Pública de México, 48(2), 179-180.

Luengo, J. (2012). La investigación y desarrollo en la industria farmacéutica: pasado, presente y futuro, Eidon. Revista de la fundación de ciencias de la salud, 37, 14-17.

Mizrahi Alvo, E. (2010). Regulación y competencia en el mercado de medicamentos: experiencias relevantes para América Latina. Ciudad de México: CEPAL.

OCDE. (2002). Manual de Frascati. Paris; FECYT.

OCDE. (2006). Manual de Oslo: Guía para la recogida e interpretación de datos sobre innovación. Oslo: Tragsa. 
OMS, OMPI, OMC. (2013). Promover el acceso a las tecnologías médicas y la innovación. Intersecciones entre la salud pública, la propiedad intelectual y el comercio. Suiza: Secretaría de la OMC.

Scuhmpeter, J. (1978). Teoría del desenvolvimiento económico. Ciudad de México: Fondo de Cultura Económica.

Tang, W. L. (2013). Revitalizing the patent system to incentivize pharmaceutical innovation: the potential of claims with means-plus-function clauses, Duke Law Journal, 62(5), 1069-1108.

Vernon, J. (2003). Price regulation, capital market imperfections, and strategic R\&D investment behavior in the pharmaceutical industry: Consequences for innovation (Tesis de doctorado). University of Pennsylvania. 